\title{
A Potent Lignan from Prunes Alleviates Inflammation and Oxidative Stress in Lithium/Pilocarpine-Induced Epileptic Seizures in Rats
}

\author{
Fadia S. Youssef ${ }^{1}\left(\mathbb{D}\right.$, Esther T. Menze ${ }^{2}$ and Mohamed L. Ashour ${ }^{1, *(\mathbb{D}}$ \\ 1 Department of Pharmacognosy, Faculty of Pharmacy, Ain Shams University, Cairo 11566, Egypt; \\ fadiayoussef@pharma.asu.edu.eg \\ 2 Department of Pharmacology and Toxicology, Faculty of Pharmacy, Ain Shams University, Abbassia, \\ Cairo 11566, Egypt; esther.menze@gmail.com \\ * Correspondence: ashour@pharma.asu.edu.eg; Tel.: +20-10-68-222-354
}

Received: 29 May 2020; Accepted: 24 June 2020; Published: 2 July 2020

\begin{abstract}
Prunus domestica L. is an edible plant that is included in the family Rosaceae and proven to possess potent anti-inflammatory and anxiolytic activity. Pinoresinol-4-O- $\beta$-D-glucopyranoside (PGu) was isolated from Prunus domestica methanol extract and its structure was determined using 1-D and 2-D NMR (one- and two-dimensional nuclear magnetic resonance). PGu was evaluated for its anticonvulsant activity using lithium/pilocarpine-induced epileptic seizures in rats. PGu displayed a notable antioxidant and anti-inflammatory activity in vitro. It ameliorates the seizures triggered by pilocarpine in a dose-dependent manner, manifested by retarding seizure onset, reducing the number of rats developing seizures, and enhancing the survival of animals after seizure exposure. PGu reduced MDA (malondialdehyde) level by $24.2 \%$ in addition to increasing catalase activity by $44.4 \%$ at $50 \mathrm{mg} / \mathrm{kg}$ b.w compared to pilocarpine-treated animals. This was confirmed by histopathological examination in which pretreatment with PGu $(50 \mathrm{mg} / \mathrm{kg}$ b.w.) attenuated neurodegeneration and seizures with no histopathological alteration in neurons of the cerebral cortex. In the immunohistochemical examination, it significantly declined the elevated Cyclooxygenase-2 (COX-2) by $40 \%$ and decreased Inducible nitric oxide synthase (iNOS) expression by $18 \%$ as expressed by the optical density. PGu revealed a pronounced fitting within the active site of 5-LOX (lipoxygenase-5) with a free binding energy $(\Delta G)$ equals to $-65.05 \mathrm{kcal} / \mathrm{mol}$. PGu could perfectly serve as a potent lead drug for the relief of epileptic seizures, which appeals to many patients owing to its natural origin.
\end{abstract}

Keywords: anti-inflammatory; epilepsy; oxidative stress; pinoresinol-4-O- $\beta$-D-glucopyranoside; pilocarpine; Prunus domestica

\section{Introduction}

Epilepsy is one of the popular neurological dysfunctions, which is characterized by the recurrent occurrence of unprovoked seizures caused by hyperneuronal activity and a sudden rush of electrical activity in the brain [1]. Unfortunately, it affects nearly 60 million people all over the globe [2]. It could be triggered by injuries, such as head injury and hypoxia, or by status epilepticus. The latter is induced by the overproduction of reactive oxygen and nitrogen species that immediately precedes oxidative stress, inflammation, and tissue damage. Basically, the vigorous liberation of cytokines as well as interleukins and their existence in the tissue is accompanied by functional impairment in the neurons. Furthermore, reactive oxygen species (ROS) and nitric oxide synthase (NOS) can contribute to neuronal damage and neuroinflammation, which are observed in status epilepticus. Increased neuronal hyperexcitability triggered by reduced Gamma aminobutyric acid (GABA) function and enhanced 
$\mathrm{N}$-methyl-D-aspartate (NMDA) receptor activity causes massive Ca++ entry in cells, which results in seizures, with subsequent generation of ROS [3,4].

Many antiepileptic synthetic agents, such as carbamazepine, oxcarbazepine, ethosuximide, felbamate, phenobarbital, as well as valproic acid and phenytoin, are present. Although they can effectively ameliorate epileptic seizures in the majority of patients, a considerable percentage, estimated by $30 \%$ to $40 \%$, still suffer from pharmacoresistance during the course of their therapy. In addition, these synthetic agents trigger undesirable side effects [5]. The plant kingdom provides an everlasting source of naturally occurring drugs and phytoconstituents that effectively participate in the amelioration of epileptic seizures. Herbal drugs have been highlighted, particularly in traditional medicine, and display significant antiepileptic results with relatively minimal side effects [6,7].

Prunus domestica L. is an edible plant that is included in the family Rosaceae. It is native to western Asia, mainly in the Caucasus area. Its edible fruits have shown a high efficacy in relieving leucorrhoea as well as controlling abnormal menstruation and combating oxidative stress owing to their high content of polyphenols. Recently, Prunus domestica has been proven to possess potent anti-inflammatory and anxiolytic activity [8].

In this context, pinoresinol-4-O- $\beta$-D-glucopyranoside, a lignan glycoside, was isolated from Prunus domestica total methanol extract. This study aimed to correlate Prunus domestica anti-inflammatory and anxiolytic activity to a compound rather than chlorogenic acid, which was previously reported to possess an anti-inflammatory effect. Pinoresinol-4-O- $\beta$-D-glucopyranoside, was evaluated for its antioxidant and anti-inflammatory activity in vitro using 2,2-diphenyl-1-picrylhydrazyl (DPPH) scavenging capacity and lipoxygenase inhibitory activity assays, respectively. Moreover, the ability of pinoresinol-4-O- $\beta$-D-glucopyranoside to counteract epileptic seizures was estimated in vivo using lithium/pilocarpine-induced status epilepticus in rats, which was further ascertained via histopathological and immunohistochemical evaluation. In addition, in silico molecular modelling was done in the 5-lipoxygenase (5-LOX) active site to confirm the anti-inflammatory activity by visualizing the possible interactions at the binding site.

\section{Materials and Methods}

\subsection{Chemicals and Drugs}

Pilocarpine hydrochloride was obtained from Alexandria Co. for pharmaceuticals and chemical industries, Egypt. $\mathrm{LiCl}$ was purchased from El-Gomhouria for trading chemicals, Cairo, Egypt. Atropine sulfate was obtained from Sigma-Aldrich Co., St. Louis, MO, USA. Spectrophotometric diagnostic kits for the assessment of malondialdhyde (MDA) level as well as catalase activity were purchased from (Biodiagnostics, Cairo, Egypt). All other consumed chemicals were of the highest grade.

\subsection{Plant Material}

Prunus domestica L. (Rosaceae) dried fruits were obtained from the Egyptian market. Identification and authentication were performed morphologically by one of the authors (M.L.A.), Associate Professor of Pharmacognosy, Faculty of Pharmacy, Ain Shams University. A voucher specimen was kept at Pharmacognosy Department, Faculty of Pharmacy, Ain Shams University, Egypt with the code PHG-P-PD-254.

\subsection{Preparation of the Plant Extract}

Dried prunes $(7 \mathrm{~kg})$ were extracted at $25^{\circ} \mathrm{C}$ with $(3 \times 7 \mathrm{~L})$ distilled methanol till exhaustion followed by filtration and evaporation under vacuum by a rotary vacuum evaporator at $40{ }^{\circ} \mathrm{C}$. A semisolid residue $(1.7 \mathrm{~kg}$ ) was obtained and then fractionated using a Diaion HP-20 packed column chromatography employing three solvent systems, which were water, methanol, and acetone. 


\subsection{Isolation and Identification of Pinoresinol-4-O- $\beta$-D-glucopyranoside}

\subsubsection{Isolation of Pinoresinol-4-O- $\beta$-D-glucopyranoside}

About $30 \mathrm{~g}$ of the methanol fraction (PMF) were further fractionated using a polyamide $6 \mathrm{~S}$ packed column using water/methanol of decreasing polarity till 100\% pure methanol was reached to give 10 main fractions. Fraction II was consequently fractionated and purified on a normal phase silica gel packed column using dichloromethane/methanol of increasing polarity, where $160 \mathrm{mg}$ of pinoresinol-4-O- $\beta$-D-glucopyranoside (PGu) were obtained with 90:10 $\left(\mathrm{CH}_{2} \mathrm{Cl}_{2} / \mathrm{MeOH}\right)$ as a solvent system.

\subsubsection{Identification of Pinoresinol-4-O- $\beta$-D-glucopyranoside}

Nuclear Magnetic Resonance Spectroscopy (NMR)

A Varian $500 \mathrm{MHz}$ AC NMR spectrometer was used to perform ${ }^{1} \mathrm{H}$ and ${ }^{13} \mathrm{C}$ (APT) $\mathrm{NMR}$ analyses using 500.13 and $125.67 \mathrm{MHz}$ as the operating frequencies, respectively. The isolated compound was solvated in deuterated dimethyl sulfoxide (DMSO) ((Deutero, Kastellaun, Germany) and $\delta$ ppm was used to express the chemical shift relative to the solvent.

Pinoresinol-4-O- $\beta$-D-glucopyranoside

It was isolated as a very pale yellow almost white powder (Figure 1$)$. Its ${ }^{1} \mathrm{H}-\mathrm{NNR}(500 \mathrm{MHz})$ and ${ }^{13} \mathrm{C}$-NNR $(125 \mathrm{MHz})$ spectral analyses in $\left(\mathrm{DMSO}-d_{6}\right)$ gave signals $(\delta \mathrm{ppm})$ that match with that previously reported in literature [9]. NMR spectral data are illustrated in Figure S1 as Supplementary Materials data.

\subsection{Evaluation of the Biological Activity In Vitro}

2.5.1. Determination of the Antioxidant Activity using Diphenyl-1-picrylhydrazyl Scavenging Capacity Assay

This was determined using the method previously reported [10,11]. Basically, $200 \mu \mathrm{L}$ of different concentrations of the isolated compound $(\mathrm{PGu})(2.5-150 \mu \mathrm{g} / \mathrm{mL})$ and $3.8 \mathrm{~mL}$ of $60 \mu \mathrm{g} / \mathrm{mL}$ of DPPH solution in methanol were mixed together. The reaction mixture was kept in the dark for $30 \mathrm{~min}$ at room temperature followed by measurement of the absorbance at $517 \mathrm{~nm}$. Trolox was used as a positive control. The DPPH${ }^{\bullet}$ : Free radical scavenging activity was determined by applying the following equation:

$$
\% \text { Inhibition }=[\mathrm{Ac}-\mathrm{As} / \mathrm{Ac}] \times 100,
$$

in which Ac: absorbance of control; As: absorbance of sample; and $\mathrm{IC}_{50}(\mu \mathrm{g} / \mathrm{mL})$ was obtained from the constructed graph. Additionally, the total antioxidant capacity (TAC) was determined for the compound and compared to Trolox. Total antioxidant activity is expressed as ascorbic acid equivalents. Values are expressed as means \pm SEM of three experiments.

\subsubsection{Determination of the Anti-Inflammatory Activity using Lipoxygenase Inhibition Assay}

Lipoxygenase (LOX) inhibitory potential of PGu was determined using the method previously reported [12,13]. Briefly, a mixture of $1 \mathrm{~mL}$ of $0.1 \mathrm{M}$ of sodium borate buffer and $10 \mu \mathrm{L}$ of $8000 \mathrm{U} / \mathrm{mL}$ of soybean LOX was kept with $10 \mu \mathrm{L}$ of different concentrations of the isolated compound (PGu) $(20-280 \mu \mathrm{g} / \mathrm{mL})$ for $5 \mathrm{~min}$ at room temperature in a 1-mL cuvette. This was followed by the addition of $10 \mu \mathrm{L}$ of $10 \mathrm{mmol}$ of linoleic acid and then the absorbance was measured at $234 \mathrm{~nm}$. Lipoxygenase inhibition was determined from the following equation:

$$
\text { \%Inhibition }=[\text { Ac-As/Ac }] \times 100,
$$


in which Ac: absorbance of control; As: absorbance of sample; and $\mathrm{IC}_{50}(\mu \mathrm{g} / \mathrm{mL})$ was obtained from the constructed graph. Trolox was used as a positive control. Values are expressed as means \pm SEM of three experiments.

\subsection{Evaluation of the Anti-Epileptic Activity In Vivo}

\subsubsection{Animals and Animal Treatment}

Male Wister rats (150-200 g) were purchased from Nile Company for Pharmaceutical and Chemical industries, Egypt. Approval of the experimental protocol was achieved by the Bioethical and Research committee of Faculty of Pharmacy, Ain Shams University, Cairo, Egypt (Permit no.56). Rats were kept under standard conditions of temperature $\left(24 \pm 5^{\circ} \mathrm{C}\right)$ and relative humidity $(55 \pm 5 \%)$ with a light/dark cycle of $12 \mathrm{~h}$ each. They were allowed to freely access standard laboratory water as well as laboratory pellets. Moreover, the animals were accommodated to the updated environmental conditions in the housing of the research facility before conducting the experimental study for one week.

\subsubsection{Experimental Design of Status Epilepticus in a Rat Model}

The animals were distributed randomly into 4 groups, each with 6 animals as follows. In group 1 (control group), animals were treated with the respective vehicles only, administered i.p. with saline, the vehicle of pilocarpine and lithium, and orally administered $0.5 \%$ CMC, the vehicle of PGu. However, animals in group 2 were treated with lithium chloride $(\mathrm{LiCl}) /$ pilocarpine in a dose of $30 \mathrm{mg} / \mathrm{kg}$ b.w. In group 3, lithium chloride $(\mathrm{LiCl}) /$ pilocarpine-treated animals orally received $25 \mathrm{mg} / \mathrm{kg} \mathrm{b.w.} \mathrm{PGu.}$ Meanwhile, in group 4, lithium chloride $(\mathrm{LiCl}) /$ pilocarpine-treated animals orally received $50 \mathrm{mg} / \mathrm{kg} \mathrm{b.w}$. PGu. The protocol was carried out as follows: Animals received $\mathrm{LiCl}$ injection ( $3 \mathrm{mEq} / \mathrm{kg}$ b.w., i.p.). After $24 \mathrm{~h}$, the animals were injected with pilocarpine hydrochloride at a dose of $30 \mathrm{mg} / \mathrm{kg} \mathrm{b.w}$. In addition, atropine sulfate at a dose of $1 \mathrm{mg} / \mathrm{kg}$ b.w. was i.p. administered 30 min before pilocarpine treatment to decrease the cholinergic adverse effects that can occur peripherally. In groups 3-4, the animals were administered two doses of the respective drug $12 \mathrm{~h}$ apart, with the second dose administered one hour prior to pilocarpine administration. All the groups administered with pilocarpine were monitored for two hours to detect any behavioral alteration. Seizures scores were assessed according to Racine scale. Briefly, in stage 1, face and vibrissae twitching, ear rubbing with forepaws, and chewing occur. In stage 2, head nodding and unilateral limb clonus is predominant. While in stage 3 , limb clonus, and mild whole-body convulsions commonly occur. In stage 4 , rearing with bilateral forelimb clonus, tail hypertension, lockjaw, and whole-body convulsions mostly happen. Finally, in stage 5, rearing with whole-body convulsions and falling down with general body rigidity occur [14]. Status epilepticus onset, latency period, and percentages of animals that showed status epilepticus and mortality rates were recorded. Then, each group was splitted into two sets after seizure evaluation, one set for histopathological and immunohistochemical evaluation. However, the other set was used to assess the oxidative stress markers $24 \mathrm{~h}$ after status epilepticus induction.

\subsubsection{Evaluation of the Biochemical Parameters}

\section{Estimation of Tissue Malondialdehyde (MDA)}

Malondialdehyde (MDA) is considered the main thiobarbituric acid-reactive species (TBARS) and it is indicative of lipid peroxidation. MDA was assessed following the method previously described [15]. Briefly, $0.5 \mathrm{~mL}$ of the tissue homogenate, $3 \mathrm{~mL}$ of $1 \% \mathrm{O}$-phosphoric acid solution, and $1 \mathrm{~mL}$ of the $0.6 \%$ TBA solution were added, mixed, and then placed in a boiling water bath for $45 \mathrm{~min}$. After cooling, the pink color formed was extracted by $n$-butanol and detected at 2 wave lengths (535 $\mathrm{nm}$ and $520 \mathrm{~nm}$ ), and then the absorbance difference was calculated. MDA levels were expressed as nanomoles per gram tissue (nmol/g tissue). 


\section{Estimation of Catalase Activity}

This was estimated following the instructions of the kit supplied by (Biodiagnostics, Cairo, Egypt) (Aebi, 1984). Briefly, catalase in the tested samples was allowed to react with a measured amount of $\mathrm{H}_{2} \mathrm{O}_{2}$. Stopping of the reaction was done after exactly one minute using a catalase inhibitor. In the presence of peroxidase (HRP), the remaining $\mathrm{H}_{2} \mathrm{O}_{2}$ reacted with 3,5-dichloro-2 hydroxybenzene sulfonic acid (DHBS) and 4-aminophenazone (AAP) to form a chromophore with a color intensity inversely proportional to the amount of catalase in the original sample and its absorbance was measured at $510 \mathrm{~nm}$.

\subsubsection{Histological Examination}

Samples from rat brains in the various groups were taken by autopsy followed by their fixation for $24 \mathrm{~h}$ in $10 \%$ formol saline. Tap water was used to wash the samples followed by their dehydration via the use of serial dilutions of different alcohol solutions, which were methanol, ethanol, and absolute ethanol. Then, xylene was used to clear the specimens, then embedded in paraffin and kept for $24 \mathrm{~h}$ at $56{ }^{\circ} \mathrm{C}$ in a hot oven. Sledge microtome was used to make sections of $4-\mu \mathrm{m}$ thickness in the prepared paraffin tissue blocks. The prepared tissue sections were gathered on glass slides then deparaffinized and stained with hematoxylin and eosin ( $\mathrm{H} \& \mathrm{E})$ to be examined routinely using a light electric microscope [16]. Scoring was performed by a blinded histologist, using an arbitrary scale, depending on the size and frequency of the lesion.

\subsubsection{Immunohistochemical Expression of COX-2 and iNOS Proteins}

Tissue sections of a 4- $\mu \mathrm{m}$ thickness were deparaffinized using xylene, then dehydrated using alcohol and heated for $5 \mathrm{~min}$ in citrate buffer to retrieve antigen. Then, the manufacturer's protocol was used to conduct immunohistochemistry staining. Basically, cyclooxygenase-2 (COX-2) monoclonal antibody (Thermo Fisher Scientific, Hemel Hempstead UK, Catalog number RB-9072-P) or iNOS (inducible nitric oxide synthase) (Thermo Fisher Scientific, Hemel Hempstead, UK, Catalog number: RB-9242-R7) or mouse anti-human GFAP (Glial fibrillary acidic protein) (Thermo Fisher Scientific, Hemel Hempstead UK) were incubated separately with the deparaffinized tissue sections after being treated for $20 \mathrm{~min}$ with $0.03 \% \mathrm{H}_{2} \mathrm{O}_{2}$. The slides were washed by TBS (Tris-Hcl Buffered Saline) and then incubated with the secondary antibody that corresponded to the antigen then the slides were washed again with TBS and kept for $10 \mathrm{~min}$ in $0.02 \%$ diaminobenzidine (DAB) with $0.01 \% \mathrm{H}_{2} \mathrm{O}_{2}$. The slides were counterstained with hematoxylin and examined by a light microscope. Image analysis software (Image J, 1.48a, NIH, Rockville, Maryland, USA) was used for immunohistochemical quantitation of antibodies. For each section, determination of the optical density of stained positive cells was done through 10 different fields.

\subsubsection{Statistical Analysis}

For parametric data, one-way ANOVA was used to perform the statistical analyses, which were consequently followed by Tukey as a post-hoc test $(p<0.05)$ following the recommendations for data and statistical analyses previously described [17]. Data are represented as mean \pm S.E.M. For non-parametric data, Kruskal Wallis followed by Dunn's test was used for data analysis $(p<0.05)$. Data are presented as median and interquartile range. Mortality rates and the occurrence of seizures were analyzed using the Chi square test for dependence and presented in a contingency table. All statistical analyses and graphs were done using GraphPad Prism software (version 5.01, Inc., 2007; San Diego, CA, USA).

\subsection{Molecular Modelling Studies}

Virtual screening was performed in silico using Discovery Studio 4.5 (Accelrys Inc., San Diego, CA, USA) employing the C-docker protocol. Docking of PGu was performed on 5-lipoxygenase (5-LOX) (PDB ID 3V99, $2.48 \AA$ ), which was downloaded from the protein data bank (www.pdb.org). The free 
binding energies for the highly stable docking poses were calculated in accordance with previously described methods [18,19].

\section{Results}

\subsection{Isolation and Identification of Pinoresinol-4-O- $\beta-\mathrm{D}-$ glucopyranoside}

Prunus domestica is a rich source of polyphenolic compounds upon extraction with methanol and repeated chromatographic separation and purification. Pinoresinol-4-O- $\beta$-D-glucopyranoside (PGu) was isolated as one of its predominant constituents. The structure of PGu is illustrated in Figure 1. The structure of the isolated compound was unambiguously determined based upon ${ }^{1} \mathrm{H}$ and ${ }^{13} \mathrm{C}$ (APT) NMR analyses that matched with that previously reported in the literature [9].

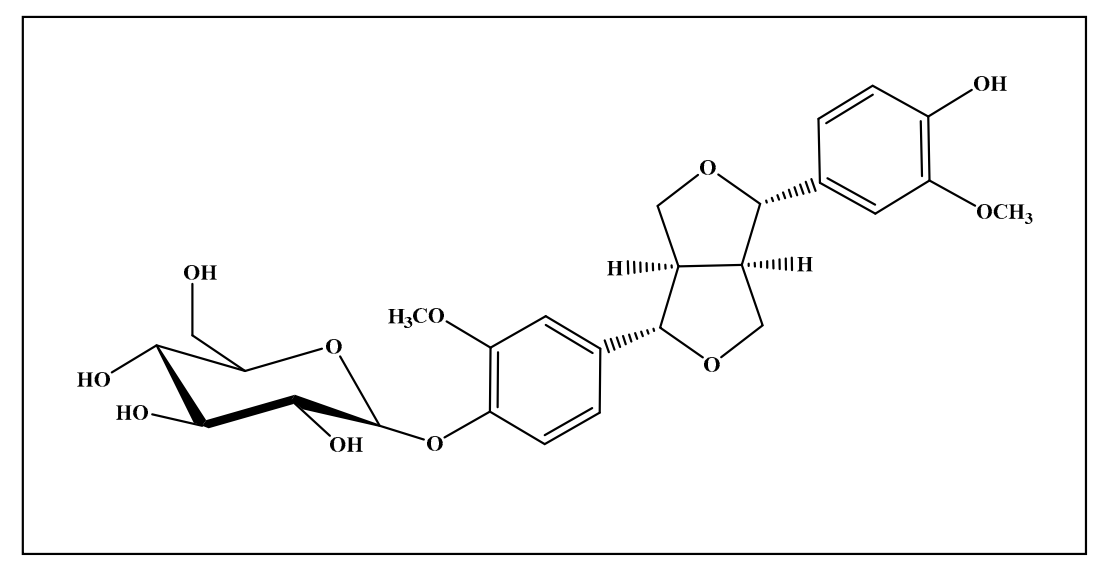

Figure 1. Pinoresinol-4-O- $\beta$-D-glucopyranoside (PGu) structure.

\subsection{Determination of the Antioxidant Activity In Vitro}

PGu antioxidant activity was determined in vitro using the most commonly used antioxidant assay, which is the diphenyl-1-picrylhydrazyl scavenging capacity assay. PGu displayed a substantial antioxidant activity, with an $\mathrm{IC}_{50}$ of $44.2 \pm 0.5 \mu \mathrm{g} / \mathrm{mL}$ (Figure $2 \mathrm{~A}$ ) and TAC of $737.7 \pm 28.9 \mu \mathrm{mol} / \mathrm{g}$ relative to Trolox, the positive control, which showed an $\mathrm{IC}_{50}$ of $4.8 \pm 0.4 \mu \mathrm{g} / \mathrm{mL}$ and TAC of $4460.1 \pm 28.9 \mu \mathrm{mol} / \mathrm{g}$.
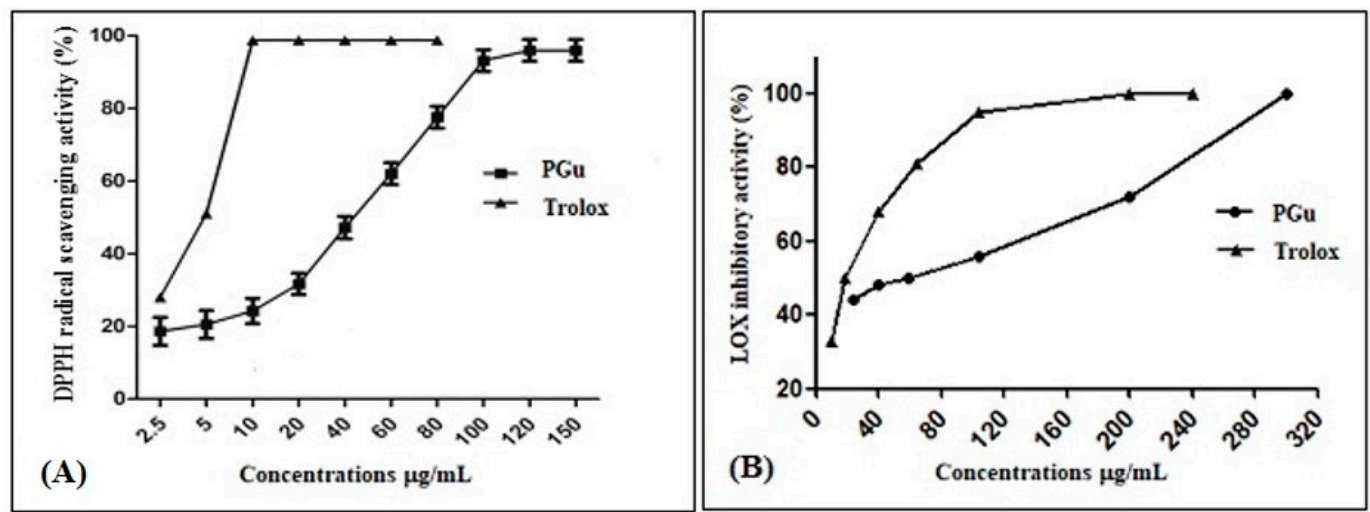

Figure 2. The radical scavenging activity of PGu (Pinoresinol-4-O- $\beta$-D-glucopyranoside) on the (2,2-diphenyl-1-picrylhydrazyl) $\mathrm{DPPH}^{\bullet}$ radicals (A) and inhibitory effects of PGu on soybean 5-lipoxygenase activity (B). 


\subsection{Determination of the Anti-Inflammatory Activity}

This was determined by applying the lipoxygenase inhibition assay, where PGu revealed promising anti-inflammatory activity, with an $\mathrm{IC}_{50}$ value of $59.05 \pm 0.5 \mu \mathrm{g} / \mathrm{mL}$ relative to Trolox, which displayed an $\mathrm{IC}_{50}$ of $18.5 \pm 0.5 \mu \mathrm{g} / \mathrm{mL}$ (Figure $2 \mathrm{~B}$ ).

\subsection{Effect of Pretreatment with Pinoresinol-4-O- $\beta$-D-glucopyranoside on LiCl/Pilocarpine-Induced Seizures}

Pilocarpine intraperitoneal injection resulted in the appearance of a series of behavioral disorders in pilocarpine-treated animals, with the appearance of stereotyped movements. These were represented by rearing, sniffing, and paw licking, which was consequently accompanied by the occurrence of seizures. However, pretreatment with PGu caused a notable amelioration in pilocarpine-induced seizures in a dose-dependent manner as evidenced by the decrease in the animal percentage that developed status epilepticus together with reduced seizure severity in addition to delayed onset of status epilepticus in a significant manner. Regarding the pilocarpine-treated group, all animals (100\%) developed seizures, with a mortality rate of 50\% after the development of status epilepticus by $24 \mathrm{~h}$. However, pretreatment with $25 \mathrm{mg} / \mathrm{kg}$ b.w. PGu slightly alleviated the severity of seizures as manifested by the reduced number of rats that developed seizures (83\%), revealing $33 \%$ mortality $24 \mathrm{~h}$ after the development of status epilepticus. In contrast, pretreatment with $50 \mathrm{mg} / \mathrm{kg}$ b.w. PGu significantly ameliorated pilocarpine-induced seizures in which only 33\% of rats developed seizures, revealing no mortality after the development of status epilepticus by $24 \mathrm{~h}$. Analysis using the Chi square test showed significant effects on the occurrence of seizures and insignificant effects on mortality rates. Pretreatment with PGu in a dose of $50 \mathrm{mg} / \mathrm{kg}$ significantly decreased the seizure duration and score and increased the seizure onset as compared to the pilocarpine-treated animals. However, PGu in a dose of $25 \mathrm{mg} / \mathrm{kg}$ did not induce significant changes. PGu given alone at a dose of 25 or $50 \mathrm{mg} / \mathrm{kg}$ b.w. had no effect on the measured biochemical parameters. The contingency table showing the effect of PGu on the occurrence of seizures and mortality rates was displayed in Table 1. Meanwhile, the results showing the seizure onset, seizure duration, as well as seizure score were represented in Figure 3.

\subsection{Effect of Pretreatment with Pinoresinol-4-O- $\beta$-D-glucopyranoside on Oxidative Stress Markers}

Pretreatment with PGu effectively ameliorated the oxidative stress damage in the cerebral cortex in a dose-dependent manner as evidenced by the reduced level of MDA with a concomitant increase in the catalase activity. Pilocarpine greatly elevated the level of lipid peroxidation in the brain, displaying a $114.7 \%$ increase in the MDA level compared to the control group, in addition to reducing the catalase activity in a notable manner, showing $35.4 \%$ reduction relative to the control group. Meanwhile, PGu at a dose of 25 and $50 \mathrm{mg} / \mathrm{kg}$ b.w. resulted in $13.3 \%$ and $24.2 \%$ decline in the MDA level, respectively, compared to the pilocarpine-treated group, in addition to increasing the catalase activity by $19.4 \%$ and $44.4 \%$, respectively, compared to pilocarpine-treated animals (Table 2 ).

Table 1. Contingency table showing the effect of PGu on the occurrence of seizures and mortality rates.

\begin{tabular}{cccccccc}
\hline & & \multicolumn{2}{c}{ Seizure Occurrence } & \multicolumn{2}{c}{ Mortality } \\
\hline Group & No of Animals and & Convulsed & Non-Convulsed & \%Convulsed & Survived & Dead $\%$ Mortality \\
\hline Control & 6 & 0 & 6 & $0 \%$ & 6 & 0 & $0 \%$ \\
Pilo + Li & 6 & 6 & 0 & $100 \%$ & 3 & 3 & $50 \%$ \\
PGu (25 $\mathbf{~ m g / k g ) ~}$ & 6 & 5 & 1 & $83 \%$ & 4 & 2 & $33 \%$ \\
PGu (50 $\mathbf{~ m g / k g ) ~}$ & 6 & 2 & 4 & $33 \%$ & 6 & 0 & $0 \%$ \\
\hline
\end{tabular}




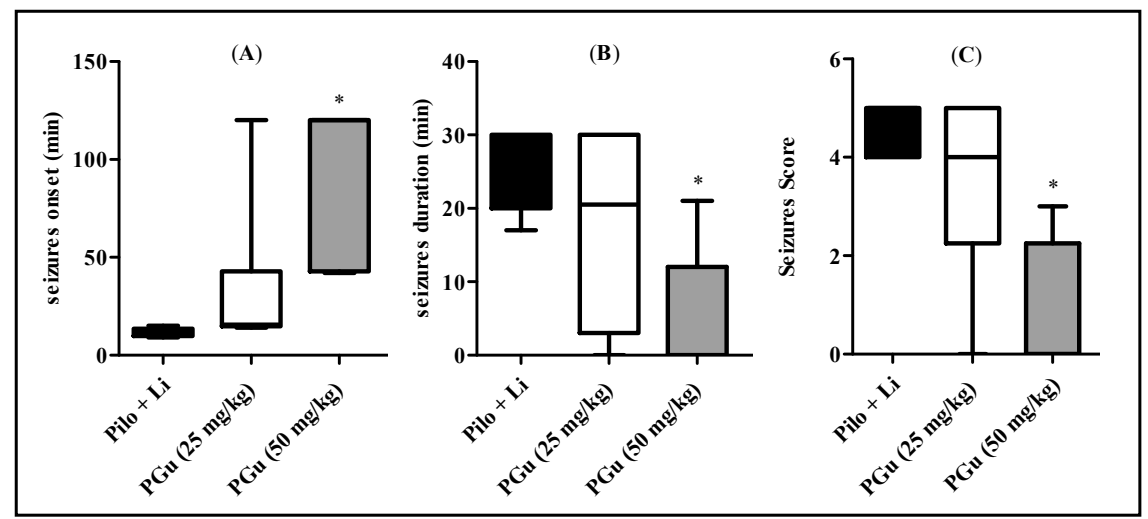

Figure 3. Effects of PGu on pilocarpine + lithium-induced seizures in rats on seizure onset (A), seizure duration (B) and seizure score (C). $\mathrm{LiCl}$ was injected i.p. in a dose of $3 \mathrm{mEq} / \mathrm{kg} \mathrm{b.w.} \mathrm{After} 24 \mathrm{~h}$, the animals were injected with pilocarpine hydrochloride in a dose of $30 \mathrm{mg} / \mathrm{kg}$ b.w. PGu was given in doses 25 and $50 \mathrm{mg} / \mathrm{kg}$ twice and the second dose was given $1 \mathrm{~h}$ before piloarpine. Data are presented as median (25th, 75th percentile) and analyzed by Kruskal-Wallis and Dunn's as a post-hoc test. The superscript * indicates significance in relation to the pilocarpine + lithium-treated group at $p<0.05$.

Table 2. Effect of PGu on the oxidative stress biomarkers (MDA) and (CAT) in pilocarpine-induced seizures in a rat model.

\begin{tabular}{|c|c|c|c|c|}
\hline Group & MDA (nmol/g tissue) & $\%$ Change & Catalase (U/g tissue) & $\%$ Change \\
\hline Control & $58.7 \pm 3.3$ & $-53.41 \% \mathrm{~b}$ & $44.6 \pm 1.9$ & $+54.86 \% \mathrm{~b}$ \\
\hline Pilo + Li & $126^{\#} \pm 6.3$ & $+114.7 \%$ a & $28.8^{\#} \pm 0.7$ & $-35.4 \%$ a \\
\hline PGu (25 mg/kg) & $109.3 \pm 5.8$ & $-13.3 \% \mathrm{~b}$ & $34.4 \pm 1.8$ & $+19.4 \% \mathrm{~b}$ \\
\hline PGu (50 mg/kg) & $95.5^{*} \pm 5.9$ & $-24.2 \% \mathrm{~b}$ & $41.6^{*} \pm 1.3$ & $+44.4 \% \mathrm{~b}$ \\
\hline
\end{tabular}

Catalase activity and MDA levels data are presented as mean $\pm \operatorname{SEM}(n=6)$ and analyzed by one-way ANOVA then Tukey as a post-hoc test. The superscripts \# and * indicate significance in relation to the control and pilocarpine + lithium-treated groups, respectively, at $p<0.05 .{ }^{\text {a }} \%$ Change relative to the control group; $\mathrm{b} \%$ Change relative to pilocarpine-treated groups, at $p<0.05$.

\subsection{Effect of Pretreatment with Pinoresinol-4-O- $\beta$-D-glucopyranoside on Neuronal Histology}

PGu decreases the neuronal damage in the cerebral cortex as supported by the histopathological examination using hematoxylin and eosin ( $\mathrm{H} \& \mathrm{E}$ staining) (Figure $4 \mathrm{~A}$ ). It was clear that animals of the control group displayed normal neurons, which are characterized by a normal alignment and structure in which the nuclei are oval or round, showing clear nucleoli, a regular distribution of chromatin, as well as transparent cytoplasm. However, pilocarpine-treated animals revealed severe neuronal damage evidenced by the appearance of nuclear pyknosis, shrinkage of the cells taking the form of a triangle, degeneration, cellular edema, and vascular congestion in the neurons of the outer and deep cerebral cortex; in addition, some nuclei appeared in a crescent form. However, pretreatment with PGu in a dose of $25 \mathrm{mg} / \mathrm{kg}$ b.w. did not show any significant neuronal protection, where the cerebral cortex showed nuclear pyknosis and degeneration in the neuronal cells. Meanwhile, it is obvious from Figure 4A that pretreatment with PGu in a dose of $50 \mathrm{mg} / \mathrm{kg}$ b.w. effectively attenuated neurodegeneration triggered by seizure induction as there was no histopathological alteration. The normal histological structure of the cerebral cortex neurons was recorded and approached that of the normal control group. Additionally, a table representing the scoring of histological changes was added to better illustrate the histological variations among the different groups (Table 3).

\subsection{Effect of Pretreatment with Pinoresinol-4-O- $\beta$-D-glucopyranoside on Neuroinflammation Manifested by} COX-2, iNOS, and GFAP Immunohistochemical Expression

Immunohistochemical staining was used to assess the expression levels of the inflammatory biomarkers COX-2 and iNOS in the cerebral neurons. The control group displayed a reduced level of immunostaining for both markers; meanwhile, pilocarpine-treated animals showed significant 
elevation in both COX-2 and iNOS by an estimated 108\% and 79\%, respectively, in its assessed optical density (OD) and 131\% and 269\%, respectively, in the area percent of stained cells (A\%) compared to the control group. PGu in a dose of $25 \mathrm{mg} / \mathrm{kg}$ b.w. insignificantly reduced the elevated COX-2 and iNOS expression by $15 \%$ and $17 \%$, respectively, as expressed by the OD and $19 \%$ and $16 \%$, respectively, as expressed in A\%. In contrast, pretreatment with PGu in a dose of $50 \mathrm{mg} / \mathrm{kg}$ b.w. significantly declined the elevated COX-2 by $40 \%$ and insignificantly decreased iNOS expression by $18 \%$ as expressed by OD and significantly reduced the A\% of both COX-2 and iNOS stained cells by $46 \%$ and $77 \%$, respectively. The immunohistochemical staining was determined via quantitative assessment of the optical density of the stained areas using the image analysis software and is represented in Figure 4B,C and Figure 5. Additionally, to better explain the potential effect of PGu on glial cells, immunohistochemical staining of the astrocyte-specific protein, glial fibrillary acid protein (GFAP), the marker of astrocytosis, was assessed (Figure 6). Pilocarpine significantly elevated the expression of GFAP compared to the control group, estimated by 1.6-fold and 91\% in its assessed OD and A\%, respectively. However, PGu at a dose of $25 \mathrm{mg} / \mathrm{kg}$ b.w. substantially reduced the elevated GFAP expression by $25 \%$ and $9.5 \%$, respectively, as expressed by the OD and A\%, respectively, relative to the Li/pilocarpine-treated group. In contrast, at $50 \mathrm{mg} / \mathrm{kg}$ b.w., PGu effectively declined the elevated GFAP expression as evidenced by its faint immunoreactivity, as revealed in Figure 7 , estimated by $41 \%$ and $37 \%$ as expressed by the OD and A\%, respectively, with respect to the Li/pilocarpine-treated group.

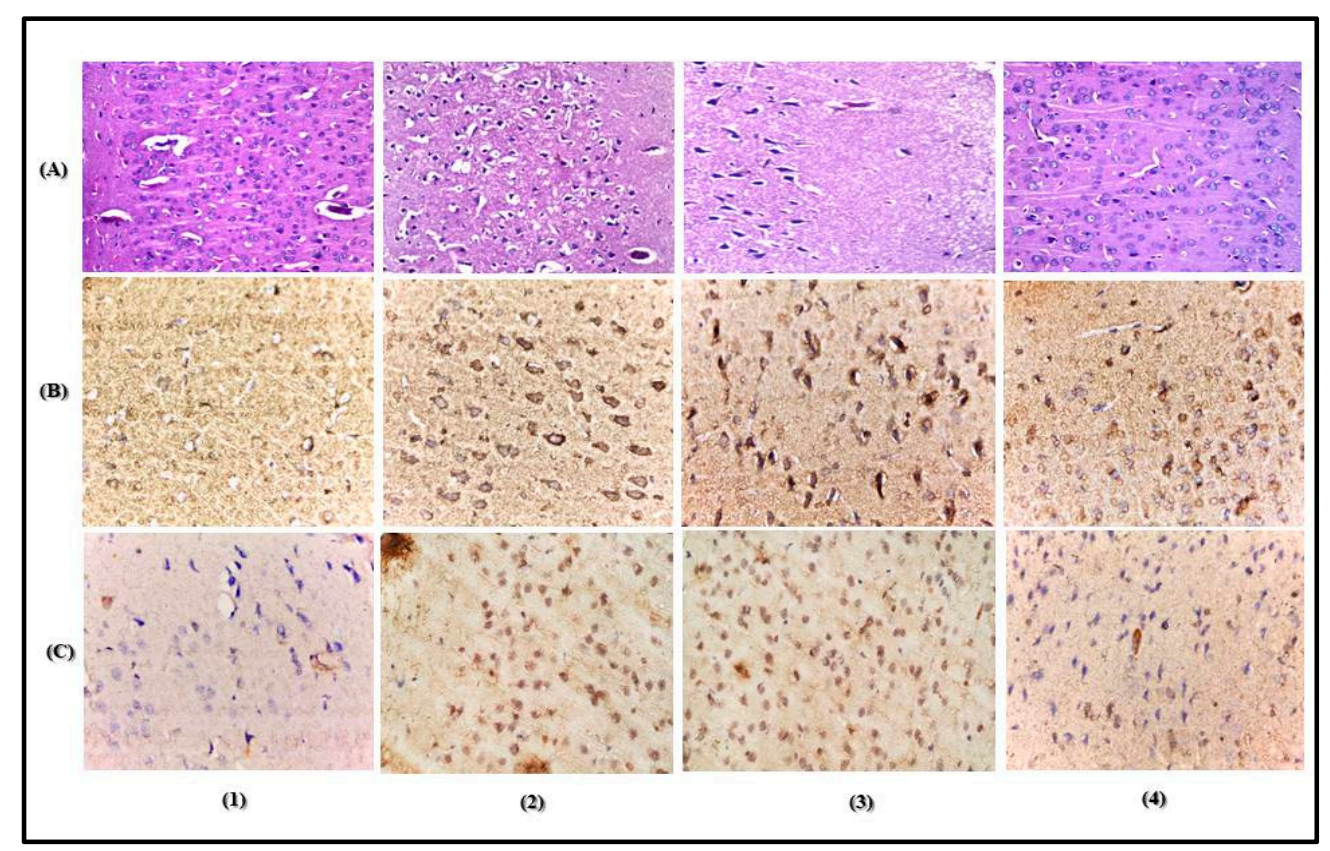

Figure 4. Photomicrographs of cerebral cortex neurons stained with hematoxylin and eosin (A), immunohistochemical staining showing Cyclooxygenase-2 (COX-2) (B) and Inducible nitric oxide synthase (iNOS) (C) expression in animals of the normal control group (1), pilocarpine status epilepticus group (2), PGu (25mg/kg b.w.) (3), and PGu (50 mg/kg b.w.) (4) pretreated groups with $40 \times$ magnification power.

Table 3. Scoring of the histological changes among different groups.

\begin{tabular}{ccccc}
\hline Histopathological Parameter & Control & LiCl/pilo & PGu (25 mg/kg) & PGu (50 mg/kg) \\
\hline Nuclear pyknosis & - & +++ & +++ & - \\
Edema & - & +++ & ++ & + \\
Vascular congestion & - & +++ & ++ & + \\
Degeneration & - & +++ & +++ & - \\
\hline
\end{tabular}

$(-)=$ null $;(+)=$ mild $;(++)=$ moderate $;(+++)=$ severe . 


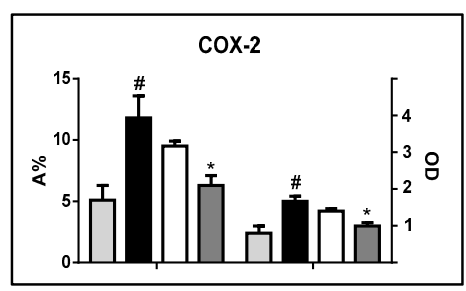

(A)

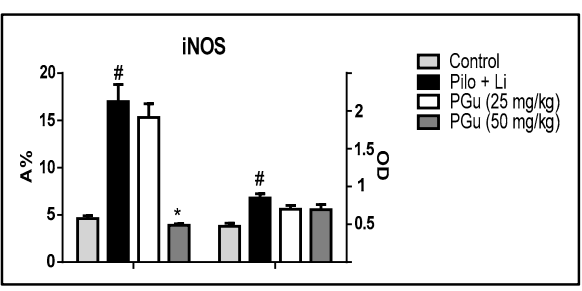

(B)

Figure 5. Quantitative image analysis for COX-2 (A) and iNOS (B) immunohistochemical staining expressed as optical densities (OD) across 7 different fields for each rat section. $\mathrm{LiCl}$ was injected at a dose of $3 \mathrm{mEq} / \mathrm{kg}$ b.w. i.p. After $24 \mathrm{~h}$, the animals were injected with pilocarpine hydrochloride at a dose of $30 \mathrm{mg} / \mathrm{kg}$ b.w. PGu was given doses of 25 and $50 \mathrm{mg} / \mathrm{kg}$ twice and the second dose was given $1 \mathrm{~h}$ before pilocarpine. Data are presented as mean \pm S.E. The superscripts \# and * indicate significance in relation to the control and pilocarpine + lithium-treated groups, respectively, at $p<0.05$.

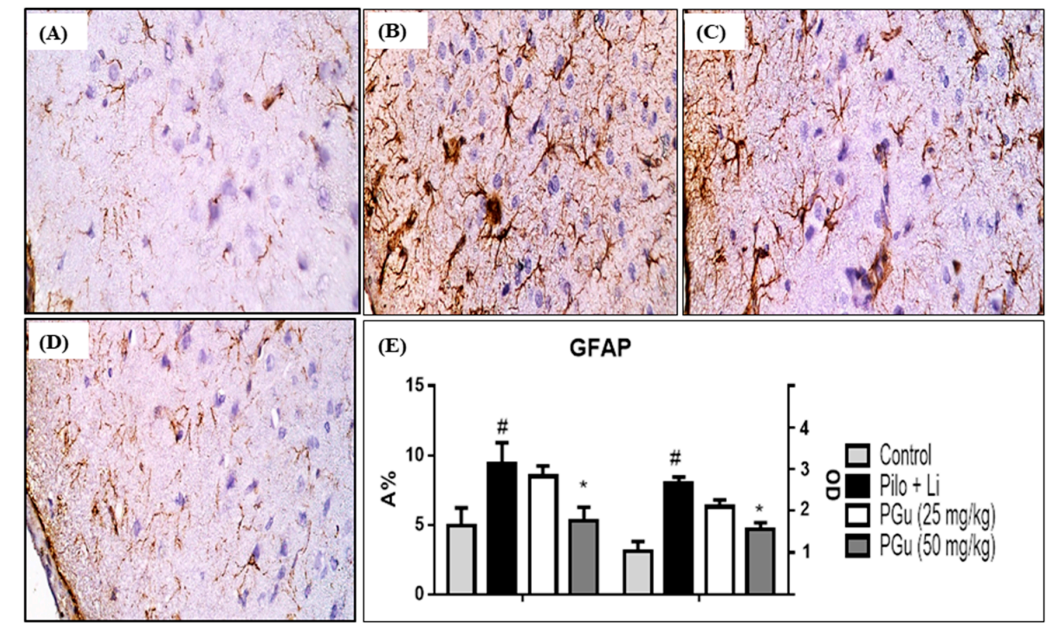

Figure 6. Photomicrographs of cerebral cortex neurons stained with immunohistochemical staining showing Glial fibrillary acidic protein (GFAP) expression in animals of the normal control group (A), pilocarpine status epilepticus group (B), PGu (25mg/kg b.w.) (C), and PGu (50 mg/kg b.w.) (D) pretreated groups with $40 \times$ magnification power while (E) shows quantitative image analysis for GFAP immunohistochemical staining expressed as optical densities (OD) across 7 different fields for each rat section. $\mathrm{LiCl}$ was injected at a dose of (mEq/kg b.w., i.p.). After $24 \mathrm{~h}$, the animals were injected with pilocarpine hydrochloride at a dose of $30 \mathrm{mg} / \mathrm{kg} \mathrm{b.w.} \mathrm{PGu} \mathrm{was} \mathrm{given} \mathrm{in} \mathrm{doses} \mathrm{of} 25$ and $50 \mathrm{mg} / \mathrm{kg}$ twice and the second dose was given $1 \mathrm{~h}$ before pilocarpine. Data are presented as mean \pm S.E. The superscripts \# and * indicate significance in relation to the control and pilocarpine + lithium-treated groups, respectively, at $p<0.05$.

\subsection{Molecular Modelling Studies}

In silico molecular modelling of PGu was done on 5-LOX, which is implicated in neuroinflammation and accompanies epileptic seizures, in an effort to confirm the in vitro as well as the in vivo anti-inflammatory potential of PGu. PGu revealed a pronounced fitting within the active site of 5 -LOX as displayed from the value of its binding free energy $(\Delta \mathrm{G})$ of $-65.05 \mathrm{kcal} / \mathrm{mol}$, exceeding that of nordihydroguaiaretic acid (NDGA), a potent anti-inflammatory agent, which revealed $(\Delta G)$ of $-61 \mathrm{kcal} / \mathrm{mol}$. Two-dimensional and 3-D binding modes of PGu within 5-LOX's active pocket are represented in Figure 6. PGu effectively inhibits 5-LOX by binding to its active sites tightly through the formation of three conventional hydrogen bonds between the hydroxyl functional groups in the glucose moiety and the ALA:672 and VAL:671 amino acid residues existing at the active site in addition to the formation of $\pi$-bond between the aromatic benzene ring and HIS:367 residue. Besides, many Van der Waals interactions occur between the different functional groups containing oxygen in the 
aglycones portion of PGu and HIS:600, GLY:174, ALA:672, ILE:406, as well as GLN:363 amino acid residues at the active sites. Furthermore, a strong metal acceptor bond formed between the molecule and iron existing in the active site, Fe2071. Meanwhile, nordihydroguaiaretic acid (NDGA) forms four H-bonds with VAL: 671, ALA: 672, and THR: 3 64; two $\pi$-bonds with LEU: 607 and ILE: 406; in addition to the strong metal acceptor bond formed between the molecule and iron existing in the active site, Fe2071. Thus, it seems that the metal acceptor bond with Fe2071, and the H- bonds with ALA: 672 and VAL: 671 are crucial for effective enzyme inhibition, as illustrated in Figure 7.

\section{Discussion}

The induction of seizures utilizing pilocarpine combined with lithium has been widely accepted as an animal model possessing many criteria of human epilepsy manifested by an electroencephalographic pattern with the consequent behavioral as well as morphological pattern. When pilocarpine is intraperitoneally injected, it triggers signs of status epilepsy that seriously damage neurons and cause excitotoxic damage [20]. Oxidative stress and inflammation are highly correlated to the occurrence of convulsions, which represents the signs of certain types of epilepsy [21-23].

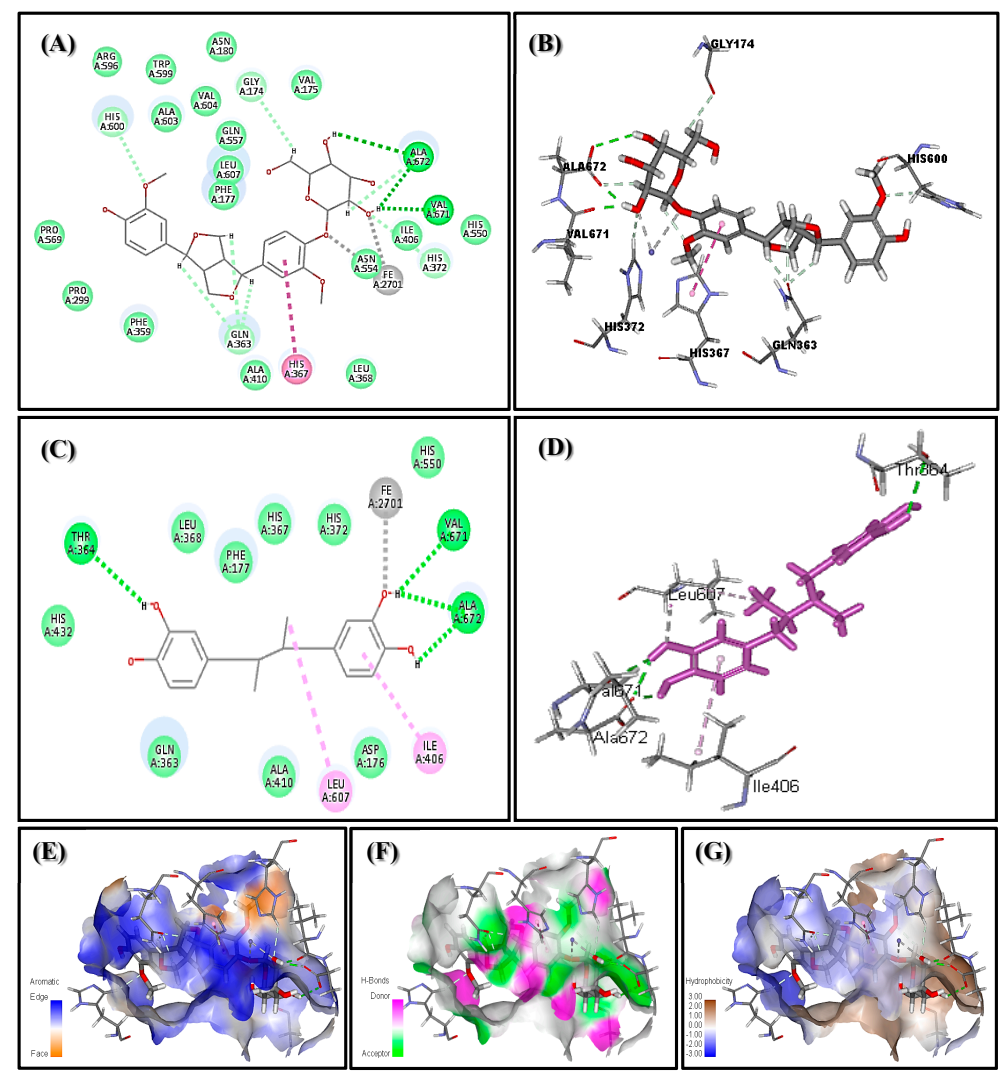

Figure 7. 2-D (A) and 3-D (B) binding modes of PGu and 2-D (C) and 3-D (D) binding modes of nordihydroguaiaretic in 5-lipoxygenase (5-LOX) active sites and the presence of PGu in the active pocket of the receptor showing the aromatic regions (E), regions of hydrogen bond formation (F), as well as hydrophobicity regions (G) in 5-LOX.

In the foregoing study, it was found that the pretreatment of rats with PGu, a dietary lignan glucoside obtained from the prunes of Prunus domestica, effectively ameliorates the seizures triggered by pilocarpine in a dose-dependent manner, as manifested by retardation in seizure onset, reduction in the number of rats that developed seizures with a concomitant reduction in its severity, and enhancement in the animal's survival after seizure exposure.

Besides, oxidative stress greatly worsens the state of epilepsy, accelerating its occurrence as well as its progression, and is strongly correlated with excitotoxicity [24]. Hence, antioxidants can effectively 
prohibit seizure initiation and ameliorate neuronal damage. Results showed that pretreatment of rats with PGu effectively ameliorates oxidative stress damage in the cerebral cortex in a dose-dependent manner as evidenced by the reduced level of MDA with a concomitant increase in catalase activity, which acts as a natural reactive oxygen species scavenger. PGu is a lignan having a furofuran skeleton with phenolic groups, which acts as a potent antioxidant via the scavenging of free radicals.

These results were further consolidated by the in vitro antioxidant activity assessment using the DPPH scavenging capacity assay displaying an $\mathrm{IC}_{50}$ of $44.2 \mu \mathrm{g} / \mathrm{mL}$. Additionally, lignans significantly inhibit the ability of many transition elements, exemplified by copper and iron triggering free radical chain reactions via the formation of complexes through their adjacent $-\mathrm{OH}$ moieties or extra chelating groups [25,26].

Regarding the histological manifestation of the neuronal structure, it was found that pilocarpine exerts a massive cellular destruction that effectively participates in epileptogenesis. It induces neuronal damage, as evidenced by the appearance of nuclear pyknosis, shrinkage of the cells taking the form of triangle, degeneration, and cellular edema. The pretreatment of rats with PGu alleviated neurodegeneration caused by pilocarpine, as manifested by the existence of a normal histological structure of the cerebral cortex neurons, particularly at a dose of $50 \mathrm{mg} / \mathrm{kg}$ b.w.

Besides, the pretreatment of rats with PGu counteracted the inflammation triggered by pilocarpine/Li treatment. It is noteworthy to highlight that a previous study was carried out on $\mathrm{PGu}$, and confirmed its antiviral activity versus influenza (H1N1) virus via its anti-inflammatory potential, as manifested by the prohibition of nuclear factor- $\mathrm{KB}$ stimulation, and inhibition of the p38 mitogen-activated protein kinase (MAPK) and Protein Kinase B (PKB/AKT) signaling pathways. Moreover, PGu effectively inhibited influenza A virus subtype H1N1 H1N1 virus-stimulated proinflammatory mediators' expression, comprising monocyte chemo-attractant protein 1 , interleukin (IL)-6, IL-8, and tumor necrosis factor- $\alpha$ [27]. Furthermore, it was reported that epileptogenesis is exaggerated by the continual production of proinflammatory mediators caused by chronic inflammation as well as seizures, leading to elevated excitation in neurons, nerve cell damage, and dysfunctional BBB [3]. PGu exerted a notable anti-inflammatory activity and effectively decreased the elevated COX-2 and iNOS expression in a dose-dependent manner, as manifested by the immunohistochemical expression. COX-2 is an enzyme that greatly affects nerve cell damage caused by seizures via oxidative stress, and excitotoxicity caused by glutamate or by prostaglandins' neurotoxic potential. COX-2 inhibitors are considered as potent therapeutic approaches in the alleviation of inflammatory neurodegenerative pathways [28]. Meanwhile, the expression of iNOS in sensitive brain areas greatly elevates the susceptibility to seizures [29]. PGu's anti-inflammatory potential was further supported by in vitro study, where PGu effectively inhibited 5-LOX of soybean, with an $\mathrm{IC}_{50}$ value of $59.05 \mu \mathrm{g} / \mathrm{mL}$. The anti-inflammatory potential of PGu was further confirmed using molecular modelling studies on 5-LOX's active site, which is implicated in the sparking and progression of inflammation [30]. 5-LOX is also entangled in many pathological disorders comprising convulsion as well as many neurodegenerative ailments that could be partly explained by the upregulation of iNOS expression [29]. PGu effectively inhibits 5-LOX by binding tightly to its active sites through the formation of three hydrogen bonds, $\pi$-bond, many Van der Waals interactions, and a metal acceptor bond at the active sites (Figure 7). This confirms the anti-inflammatory activity of PGu and its role, in turn, in alleviating convulsions and neuroinflammation associated with epilepsy. It is worthy to highlight that many lignans exerted a promising neuroprotective potential, where (+)-pinoresinol and herpetol displayed potent inhibitory effects on the cell death of the glutamate-induced HT22 (Mouse Hippocampal Neuronal Cell Line) cells and on NO production in (lipopolysaccharide) LPS-induced mouse macrophages RAW264.7 cells in a dose-dependent manner. They exerted a protective behavior estimated by $86.0 \%$ and $83.9 \%$ inhibition versus glutamate-induced cell death at a concentration of $40 \mu \mathrm{M}$, which is superior to the protective effect elicited by the positive control, Trolox [31]. Besides, neolignans represented by honokiol, obovatol, and nethylhonokiols were reported to possess neuroprotective effects that probably offer promising drug leads for the alleviation and prohibition of neuronal disease [32]. 


\section{Conclusions}

In the foregoing study, it was concluded that pinoresinol-4-O- $\beta$-D-glucopyranoside exerted potent neuroprotective activity as manifested by the alleviation of lithium/pilocarpine-induced epileptic seizures and neuroinflammation in rats. This pronounced activity greatly relies upon the antioxidant and anti-inflammatory activity of PGu as evidenced by the in vitro study, which was further consolidated by the in vivo results. The presence of the furofuran skeleton with the existence of phenolic groups mainly accounts for the promising activity of the compound. Thus, this lignan glucoside could perfectly serve as a potent therapeutic agent for the relief of epileptic seizures, which is highly welcomed by a large category of patients owing to its natural origin. However, additional clinical trials are recommended to declare the possibility of its utilization in human disorders. Future studies to better understand the pharmacokinetic-pharmacodynamic correlation are to be carried out. Additionally, other seizure models, such as (pentylenetetrazole) PTZ or (electroconvulsive seizure) ECS, are recommended to ascertain the obtained results.

Supplementary Materials: The following are available online at http://www.mdpi.com/2076-3921/9/7/575/s1. Figure S1: NMR data of Pinoresinol-4-O- $\beta$-D-glucopyranoside.

Author Contributions: F.S.Y.; Isolation and structural elucidation of the isolated compound, interpretation of the biological results, doing the in silico virtual screening as well as writing the manuscript, E.T.M.; doing the biological studies in animal models and interpretation of the results, M.L.A.; supervised the work and revised the manuscript. All authors have read and agreed to the published version of the manuscript.

Funding: This research received no external funding.

Conflicts of Interest: The authors declare no conflict of interest

\section{References}

1. Hema, B.; Bhupendra, S.; Saleem, T.M.; Gauthaman, K. Anticonvulsant effect of Drosera burmannii Vahl. Int. J. Appl. Res. Nat. Prod. 2009, 2,1-4.

2. Alexopoulos, A.V. Pharmacoresistant epilepsy: Definition and explanation. Epileptology 2013, 1, 38-42. [CrossRef]

3. González-Reyes, S.; Santillán-Cigales, J.J.; Jiménez-Osorio, A.S.; Pedraza-Chaverri, J.; Guevara-Guzmán, R. Glycyrrhizin ameliorates oxidative stress and inflammation in hippocampus and olfactory bulb in lithium/pilocarpine-induced status epilepticus in rats. Epilepsy Res. 2016, 126, 126-133. [CrossRef]

4. Lu, Y.M.; Mansuy, I.M.; Kandel, E.R.; Roder, J. Calcineurin-mediated LTD of GABAergic inhibition underlies the increased excitability of CA1 neurons associated with LTP. Neuron 2000, 26, 197-205. [CrossRef]

5. Kumar, D.; Singh, J.; Baghotia, A.; Kumar, S. Anticonvulsant effect of the ethanol extract of Caesalpinia pulcherrima (L.) Sw., Fabaceae, leaves. Rev. Bras. Farmacogn. 2010, 20, 751-755. [CrossRef]

6. Quintans-Júnior, L.; Souza, T.; Leite, B.; Lessa, N.; Bonjardim, L.; Santos, M.; Alves, P.; Blank, A.; Antoniolli, A. Phythochemical screening and anticonvulsant activity of Cymbopogon winterianus Jowitt (Poaceae) leaf essential oil in rodents. Phytomedicine 2008, 15, 619-624. [CrossRef]

7. Fathy, S.; Emam, M.; Agwa, S.A.; Zahra, F.A.; Youssef, F.; Sami, R. The antiproliferative effect of Origanum majorana on human hepatocarcinoma cell line: Suppression of NF-kB. Cell. Mol. Biol. 2016, 62, 80-84.

8. Bouayed, J.; Rammal, H.; Dicko, A.; Younos, C.; Soulimani, R. Chlorogenic acid, a polyphenol from Prunus domestica (Mirabelle), with coupled anxiolytic and antioxidant effects. J. Neurolog. Sci. 2007, 262, 77-84. [CrossRef]

9. Kim, M.-R.; Moon, H.T.; Lee, D.G.; Woo, E.-R. A new lignan glycoside from the stem bark of Styrax japonica. Arch. Pharm. Res. 2007, 30, 425-430. [CrossRef]

10. Nenadis, N.; Lazaridou, O.; Tsimidou, M.Z. Use of reference compounds in antioxidant activity assessment. J. Agric. Food Chem. 2007, 55, 5452-5460. [CrossRef]

11. Youssef, F.; Ashour, M.; Sobeh, M.; El-Beshbishy, H.; Singab, A.; Wink, M. Eremophila maculata-isolation of a rare naturally-occurring lignan glycoside and the hepatoprotective activity of the leaf extract. Phytomedicine 2016, 23, 1484-1493. [CrossRef]

12. Wu, H. Affecting the activity of soybean lipoxygenase-1. J. Mol. Graph. 1996, 14, 331-337. [CrossRef] 
13. Ashour, M.L.; Youssef, F.S.; Gad, H.A.; El-Readi, M.Z.; Bouzabata, A.; Abuzeid, R.M.; Sobeh, M.; Wink, M. Evidence for the anti-inflammatory activity of Bupleurum marginatum (Apiaceae) extracts using in vitro and in vivo experiments supported by virtual screening. J. Pharm. Pharmacol. 2018, 70, 952-963. [CrossRef] [PubMed]

14. Racine, R.J. Modification of seizure activity by electrical stimulation: II. Motor seizure. Electroencephalogr. Clin. Neurophysiol. 1972, 32, 281-294. [CrossRef]

15. Okaichi, Y.; Ishikura, Y.; Akimoto, K.; Kawashima, H.; Toyoda-Ono, Y.; Kiso, Y.; Okaichi, H. Arachidonic acid improves aged rats' spatial cognition. Physiol. Behav. 2005, 84, 617-623. [CrossRef] [PubMed]

16. Banchroft, J.; Stevens, A.; Turner, D. Theory and Practice of Histological Techniques; Churchill Livingstone: New York, NY, USA; London, UK; San Francisco, CA, USA; Tokyo, Japan, 1996.

17. Curtis, M.J.; Alexander, S.; Cirino, G.; Docherty, J.R.; George, C.H.; Giembycz, M.A.; Hoyer, D.; Insel, P.A.; Izzo, A.A.; Ji, Y. Experimental design and analysis and their reporting II: Updated and simplified guidance for authors and peer reviewers. Br. J. Pharmacol. 2018, 175, 987-993. [CrossRef] [PubMed]

18. Janibekov, A.A.; Youssef, F.S.; Ashour, M.L.; Mamadalieva, N.Z. New flavonoid glycosides from two Astragalus species (Fabaceae) and validation of their antihyperglycaemic activity using molecular modelling and in vitro studies. Ind. Crops Prod. 2018, 118, 142-148. [CrossRef]

19. Thabet, A.A.; Youssef, F.S.; El-Shazly, M.; El-Beshbishy, H.A.; Singab, A.N.B. Validation of the antihyperglycaemic and hepatoprotective activity of the flavonoid rich fraction of Brachychiton rupestris using in vivo experimental models and molecular modelling. Food Chem. Toxicol. 2018, 114, 302-310. [CrossRef]

20. Ali, A.E.; Mahdy, H.M.; Elsherbiny, D.M.; Azab, S.S. Rifampicin ameliorates lithium-pilocarpine-induced seizures, consequent hippocampal damage and memory deficit in rats: Impact on oxidative, inflammatory and apoptotic machineries. Biochem. Pharmacol. 2018, 156, 431-443. [CrossRef]

21. Aguiar, C.C.T.; Almeida, A.B.; Araújo, P.V.P.; De Abreu, R.N.D.C.; Chaves, E.M.C.; Do Vale, O.C.; Macêdo, D.S.; Woods, D.J.; Fonteles, M.M.D.F.; Vasconcelos, S.M.M. Oxidative stress and epilepsy: Literature review. Oxid. Med. Cell. Long. 2012, 2012. [CrossRef]

22. Shin, E.-J.; Jeong, J.H.; Chung, Y.H.; Kim, W.-K.; Ko, K.-H.; Bach, J.-H.; Hong, J.-S.; Yoneda, Y.; Kim, H.-C. Role of oxidative stress in epileptic seizures. Neurochem. Int. 2011, 59, 122-137. [CrossRef]

23. Golechha, M.; Sarangal, V.; Bhatia, J.; Chaudhry, U.; Saluja, D.; Arya, D.S. Naringin ameliorates pentylenetetrazol-induced seizures and associated oxidative stress, inflammation, and cognitive impairment in rats: Possible mechanisms of neuroprotection. Epilepsy Behav. 2014, 41, 98-102. [CrossRef]

24. Reynolds, I.J.; Hastings, T.G. Glutamate induces the production of reactive oxygen species in cultured forebrain neurons following NMDA receptor activation. J. Neurosci. 1995, 15, 3318-3327. [CrossRef]

25. Korkina, L. Phenylpropanoids as naturally occurring antioxidants: From plant defense to human health. Cell. Mol. Biol. 2007, 53, 15-25.

26. Youssef, F.S.; Ashour, M.L.; Ebada, S.S.; Sobeh, M.; El-Beshbishy, H.A.; Singab, A.N.; Wink, M. Antihyperglycaemic activity of the methanol extract from leaves of Eremophila maculata (Scrophulariaceae) in streptozotocin-induced diabetic rats. J. Pharm. Pharmacol. 2017, 69, 733-742. [CrossRef]

27. Li, J.; Liang, X.; Zhou, B.; Chen, X.; Xie, P.; Jiang, H.; Jiang, Z.; Yang, Z.; Pan, X. (+)-pinoresinol-O- $\beta$-D-glucopyranoside from Eucommia ulmoides Oliver and its anti-inflammatory and antiviral effects against influenza A (H1N1) virus infection. Mol. Med. Rep. 2019, 19, 563-572. [CrossRef]

28. Kunz, T.; Oliw, E.H. The selective cyclooxygenase-2 inhibitor rofecoxib reduces kainate-induced cell death in the rat hippocampus. Eur. J. Neurosci. 2001, 13, 569-575. [CrossRef]

29. Payandemehr, B.; Khoshneviszadeh, M.; Varastehmoradi, B.; Gholizadeh, R.; Bahremand, T.; Attar, H.; Bahremand, A.; Dehpour, A.R. A COX/5-LOX inhibitor licofelone revealed anticonvulsant properties through iNOS diminution in mice. Neurochem. Res. 2015, 40, 1819-1828. [CrossRef]

30. Sobeh, M.; Mahmoud, M.F.; Petruk, G.; Rezq, S.; Ashour, M.L.; Youssef, F.S.; El-Shazly, A.M.; Monti, D.M.; Abdel-Naim, A.B.; Wink, M. Syzygium aqueum: A polyphenol-rich leaf extract exhibits antioxidant, hepatoprotective, pain-killing and anti-inflammatory activities in animal models. Front. Pharmacol. 2018, 9. [CrossRef] 
31. In, S.-J.; Seo, K.-H.; Song, N.-Y.; Lee, D.-S.; Kim, Y.-C.; Baek, N.-I. Lignans and neolignans from the stems of Vibrunum erosum and their neuroprotective and anti-inflammatory activity. Arch. Pharm. Res. 2015, 38, $26-34$. [CrossRef]

32. Yang, E.-J.; Lee, J.-Y.; Park, S.-H.; Lee, T.; Song, K.-S. Neuroprotective effects of neolignans isolated from Magnoliae cortex against glutamate-induced apoptotic stimuli in HT22 cells. Food Chem. Toxicol. 2013, 56, 304-312. [CrossRef]

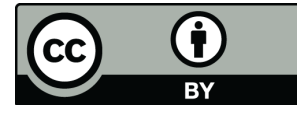

(C) 2020 by the authors. Licensee MDPI, Basel, Switzerland. This article is an open access article distributed under the terms and conditions of the Creative Commons Attribution (CC BY) license (http://creativecommons.org/licenses/by/4.0/). 